Article

\title{
Effect of Acid/Ethanol Ratio on Medium Chain Carboxylate Production with Different VFAs as the Electron Acceptor: Insight into Carbon Balance and Microbial Community
}

\author{
Shuai Bao ${ }^{1}$, Qingyan Wang ${ }^{1}$, Panyue Zhang ${ }^{1,2 * * \mathbb{D}}$, Qi Zhang ${ }^{1}$, Yan Wu ${ }^{2}$, Fan Li ${ }^{1}$, Xue Tao ${ }^{1}$, \\ Siqi Wang ${ }^{1}$, Mohammad Nabi ${ }^{1}$ and Yazhou Zhou ${ }^{1}$ \\ 1 College of Environmental Science and Engineering, Beijing Forestry University, Beijing 100083, China; \\ baoshuai16@163.com (S.B.); Wangqingyan@bjfu.edu.cn (Q.W.); zzzqi_1357@163.com (Q.Z.); \\ lipanlove1987@126.com (F.L.); taoxuegz@163.com (X.T.); wsq921029@163.com (S.W.); \\ nabiforester@gmail.com (M.N.); 17888838820@163.com (Y.Z.) \\ 2 School of Environmental and Chemical Engineering, Chongqing Three Gorges University, \\ Chongqing 404632, China; wuyan19850827@hotmail.com \\ * Correspondence: panyue_zhang@bjfu.edu.cn; Tel.: +86-15001255497
}

Received: 18 June 2019; Accepted: 26 September 2019; Published: 29 September 2019

\begin{abstract}
Medium chain carboxylates (MCCs) are important precursors for biodiesel production. Using chain elongation to produce MCCs is an emerging bioenergy technology. In this study, batch tests were conducted to investigate fermentative MCC production through chain elongation from acetate, propionate, $n$-butyrate, and ethanol. The effect of the acid/ethanol ratio on MCC production by mixed culture was investigated. Better MCC production, especially $n$-caproate production, was achieved at optimal acid/ethanol ratios of 1:4, 1:3, and 1:2 with acetate, propionate, and $n$-butyrate as the electron acceptor, respectively. The $n$-caproate concentration was high, up to $41.54 \mathrm{mmol} / \mathrm{L}$, and the highest $n$-caproate production efficiency was $57.96 \%$ with the $n$-butyrate/ethanol ratio of 1:2. The higher concentration of ethanol might stimulate the growth of chain elongation bacteria to promote chain elongation. The highest MCC production efficiency with different electron acceptors corresponded to less carbon loss and a higher chain elongation degree. In addition, with the optimal acid/ethanol ratio, the substrate was maximally utilized for chain elongation. The microbial community analysis confirmed the carbon balance analysis with the maximum relative abundance of $52.66-60.55 \%$ of the $n$-caproate producer Clostridium_sensu_stricto_12 enriched by the optimal acid/ethanol ratios with different volatile fatty acids (VFAs) as electron acceptors.
\end{abstract}

Keywords: chain elongation; acid/ethanol ratio; electron acceptor; MCC production; carbon balance; microbial community

\section{Introduction}

The energy crisis is a noticeable problem in the world. Numerous countries are finding new energies to cope with the extreme consumption of fossil fuels. Anaerobic digestion with methane output as the main energetic component has been studied by many researchers [1-5]. However, the lower energy density and economic value of methane limits its practical application [6]. Thus, in recent years, the renewable biochemical production platform called the carboxylate platform has been proposed and caught the attention of many researchers $[7,8]$. The carboxylate platform aims to produce carboxylic acids from biomass fermentation, which is of greater economic value than methane. These products include the intermediate fermentation products of biomass, volatile fatty acids (VFAs), 
such as acetate (C2), propionate (C3), and $n$-butyrate (C4), as well as their chain elongation products, medium-chain carboxylates (MCCs), such as $n$-valerate (C5), $n$-caproate (C6), $n$-heptanoate (C7), and $n$-caprylate (C8). The MCCs can be used as antimicrobials and corrosion inhibitors, or as precursors of many important biochemicals, including biodiesel and bioplastic $[9,10]$ with higher added value or higher energy density than methane and VFAs [11]. Therefore, converting VFAs into MCCs is beneficial for the maximum utilization of energy in biomass wastes.

As an emerging bioenergy technology in the carboxylate platform, carbon chain elongation has become more and more attractive in environmental research and industrial application [12-14]. The chain elongation of short-chain carboxylates (SCCs) to MCCs commonly occurs via a reverse $\beta$ oxidation pathway with ethanol as a source of carbon, energy, and reducing equivalents $[15,16]$. Steinbusch et al. [17] suggested that not only the nature of the electron donor, but also the ratio of the electron donor to electron acceptor might influence the production and component structure of chain elongation products. Therefore, several studies have been completed on the effect of the acetate/ethanol ratio on the chain elongation to achieve a higher MCC production with $n$-caproate as the targeted production. Yin et al. [18] conducted batch tests at different acetate/ethanol ratios (8:2, 7:3, 5:5, 3:7, 2:8) with a pure chain elongation bacteria culture, and found that the highest $n$-caproate concentration of $3.11 \mathrm{~g} / \mathrm{L}$ was achieved at an acetate/ethanol ratio of 3:7. Liu et al. [19] evaluated an acetate/ethanol ratio from 2:1 to $1: 3$ and showed that with an acetate/ethanol ratio of 1:3, the highest $n$-caproate concentration of $3.03 \mathrm{~g} / \mathrm{L}$ was achieved with an unacclimatized inoculum. The inoculums may also influence the optimal acetate/ethanol ratio.

On the other hand, the natural fermentation broth of biomass is normally a mixture of VFAs, mainly composed with acetate, propionate, $n$-butyrate, and so on [20-22], therefore, the optimization of the acid/ethanol ratio on a mixed chain elongation fermentation with several VFAs as the electron acceptor is important. The usage of different single acids provides a diversity of MCC products [23], which indicates a significantly varied production and component structure of chain elongation products with different VFAs as the electron acceptor corresponding to different acid/ethanol ratios. However, there is little research aimed at investigating the effect of propionate or the $n$-butyrate/ethanol ratio on the $n$-caproate production. Ge et al. [24] proposed that an $n$-butyrate/ethanol ratio of 1:2 was an optimum ratio, however, this is only a speculation without experimental verification. In addition, a systematic evaluation of the effect of the acid/ethanol ratio on the mixed culture elongation reaction with different VFAs as the electron acceptor is still needed.

Therefore, in this paper, the acid/ethanol ratios of 2:1,1:1,1:2, 1:3, and 1:4 with acetate, propionate, and $n$-butyrate as the electron acceptor was studied under an open culture. The $n$-caproate was selected as the target product because of its high production and the high proportion of even-carbon substrates in the fermentation broth [12]. The aims of this paper were to investigate the performance of chain elongation at different acid/ethanol ratios with different single acids as the electron acceptor. According to the experimental results, the carbon balance during chain elongation was analyzed and the optimal acid/ethanol ratio for different electron acceptors was estimated. The microbial community analysis was conducted to explore the effect of the ethanol ratio on the enrichment of functioned bacteria with different electron acceptors and to further clarify the mechanism of community variation. This research provides a novel insight of the acid/ethanol ratio effect on chain elongation with diverse electron acceptors under mixed culture, which is beneficial for efficient MCC production, improving economic efficiency, and the sustainability of biomass utilization.

\section{Materials and Methods}

\subsection{Open Culture Inocula}

Initial inoculation sludge was the granular sludge from an internal circulation reactor for anaerobic fermentation of high concentration organic wastewater in Beijing. The inoculation sludge was then acclimatized for chain elongation at $35^{\circ} \mathrm{C}$ for almost two months. Acetate and ethanol were added as the electron acceptor and donor in an acetate/ethanol molar ratio of 1:3 with an initial addition of 
$50 \mathrm{mmol} / \mathrm{L}$ acetate and $150 \mathrm{mmol} / \mathrm{L}$ ethanol, and the $n$-butyrate was the main product. After that, the inocula were centrifuged at $11,600 \mathrm{~g}$ for $15 \mathrm{~min}$. The supernatant was discarded to avoid interference to the substrate, and the solid was used as the open culture inocula.

\subsection{Medium}

The medium for batch chain elongation in each bottle contained $\sim 100 \mathrm{~mL}$ working solution with different concentrations of VFAs and ethanol as substrates, including pure acid $(0.3134,0.4845$, and $0.5484 \mathrm{~mL}$ for acetate, propionate, and $n$-butyrate, respectively), pure ethanol $(0.1751-1.4008 \mathrm{~mL}$ for different acid/ethanol ratios), and $100 \mathrm{~mL}$ mineral stock. The mineral stock contained $3600 \mathrm{mg} / \mathrm{L}$ $\mathrm{NH}_{4} \mathrm{H}_{2} \mathrm{PO}_{4}, 600 \mathrm{mg} / \mathrm{L} \mathrm{MgCl} 2,150 \mathrm{mg} / \mathrm{L} \mathrm{KCl}, 200 \mathrm{mg} / \mathrm{L} \mathrm{CaCl} 2 \cdot 2 \mathrm{H}_{2} \mathrm{O}, 4000 \mathrm{mg} / \mathrm{L} \mathrm{K}_{2} \mathrm{CO}_{3}$, and $1 \mathrm{~mL}$ trace element solution [17]. The initial acid (acetate, propionate, and $n$-butyrate, respectively) concentration was set as $60 \mathrm{mmol} / \mathrm{L}$. In addition, $1 \mathrm{~g} / \mathrm{L}$ yeast extract was added to improve the growth of MCC-producing bacteria [25]. Then, $10 \mathrm{~g} / \mathrm{L}$ 2-Bromoethanesulfonate (2-BrES) was added to inhibit acetoclastic methanogenesis [26].

\subsection{Batch Chain Elongation Experiments}

Batch experiments were conducted in $300 \mathrm{~mL}$ glass bottles with $100 \mathrm{~mL}$ working volume and $200 \mathrm{~mL}$ headspace. Fifteen experimental groups were implemented using acetate, propionate, and $n$-butyrate as the electron acceptor and ethanol as the electron donor with the acid/ethanol molar ratios of 2:1,1:1,1:2,1:3, and 1:4, respectively, with the initial ethanol concentrations of 30, 60, 120, 180, and $240 \mathrm{mmol} / \mathrm{L}$, respectively. The initial inocula/substrate ratio was 1:4 based on volatile solids (VS). Thus, each bottle contained $100 \mathrm{~mL}$ medium and inoculum sludge from 7.48 to $24.52 \mathrm{~mL}$, respectively. After inoculation, the system $\mathrm{pH}$ was adjusted to $6.5-7.0$ with $2 \mathrm{~mol} / \mathrm{L} \mathrm{NaOH}$ or $\mathrm{HCl}$, and the bottles were flushed with nitrogen gas for $3 \mathrm{~min}$ to remove oxygen before fermentation. All bottles were incubated at $35{ }^{\circ} \mathrm{C}$ in a rotating shaker with a rotation speed of $140 \mathrm{r} / \mathrm{min}$ for $12 \mathrm{~d}$. Every day, $2 \mathrm{~mL}$ liquid was taken out for the analysis of $\mathrm{pH}$ change, substrate degradation, and MCC formation. All experiments were performed in duplicate. The reported results were the average values with standard deviations.

\subsection{Chemical Analysis}

The $\mathrm{pH}$ of the liquid samples was measured by an on-line $\mathrm{pH}$ meter M200 with a sensor of InPro3250i/SG/120 (Mettler Toledo, Switzerland). The liquid sample was centrifuged at 11,000 $g$ for $15 \mathrm{~min}$, and the supernatant was diluted with ultrapure water to $10 \%(\mathrm{v} / \mathrm{v})$ for fatty acid analysis. Fatty acids and ethanol were analyzed by gas chromatography (GC-2018, Schimadzu (China) Co., China) with a WondaCap-WAX column ( $30 \mathrm{~m} \times 0.25 \mathrm{~mm} \times 0.25 \mu \mathrm{m}$, Schimadzu, China) and a flame ionization detector (FID). Then, $1 \mathrm{~mL}$ filtered aqueous samples $(0.45 \mu \mathrm{m}+0.22 \mu \mathrm{m})$ were placed in a vial together with $100 \mu \mathrm{L}$ freshly prepared 3\% phosphoric acid. Prepared sample of $0.5 \mu \mathrm{L}$ were injected by an auto injector (AOC-20i, Schimadzu, China) at $250^{\circ} \mathrm{C}$ with a split ratio of 35 . The oven temperature was held at $90^{\circ} \mathrm{C}$ for $1 \mathrm{~min}$, increased by $10^{\circ} \mathrm{C} / \mathrm{min}$ from 90 to $240{ }^{\circ} \mathrm{C}$, and then was kept at $240{ }^{\circ} \mathrm{C}$ for $10 \mathrm{~min}$. The carrier gas was nitrogen with a column flow rate of $1.6 \mathrm{~mL} / \mathrm{min}$. FID temperature was set at $250{ }^{\circ} \mathrm{C}$, and the flow rate of hydrogen and synthetic air was set as 40 and $400 \mathrm{~mL} / \mathrm{min}$, respectively.

\subsection{High Throughput Sequencing of the Microbial Community}

To analyze the composition of the microbial community during chain elongation fermentation, the centrifuged solids of the sludge samples were stored at $-80^{\circ} \mathrm{C}$ before DNA extraction. The sludge samples were collected at the end of the rapid reaction phase in the different chain elongation systems. Microbial DNA extraction was performed using the E.Z.N.A. soil DNA kit (Omega Bio-tek, Norcross, GA, USA). The V4-V5 region of the bacteria $16 \mathrm{~S}$ ribosomal RNA gene were amplified by PCR using primers 515F (5'-GTGCCAGCMGCCGCGG-3') and 907R (5'-CCGTCAATTCMTTTRAGTTT-3') [19]. PCR reactions were performed in triplicate with the $20 \mu \mathrm{L}$ mixture containing $5 \times$ FastPfu Buffer of $4 \mu \mathrm{L}, 2.5 \mathrm{mmol} / \mathrm{L}$ dNTPs of $2 \mu \mathrm{L}$, each primer $(5 \mu \mathrm{mol} / \mathrm{L})$ of $0.8 \mu \mathrm{L}$, FastPfu Polymerase of $0.4 \mu \mathrm{L}$, and template DNA of $10 \mathrm{ng}$. After purification and quantification of PCR products, amplicon sequencing 
was conducted using an Illumina MiSeq PE250 platform (Shanghai Majorbio Bio-pharm Technology Co. Ltd., Shanghai, China) [27].

\subsection{Evaluation of Chain Elongation Performance}

When the chain elongation reaction reached a stable phase, several metrics were used to evaluate the effect of chain elongation, MCC production, and carbon balance with different electron acceptors at different acid/ethanol ratios.

The total MCC production efficiency is the ratio of the carbon equivalent quantity (Ceq) of the total MCCs produced to that of the initial carbon source added, which is calculated as Equation (1) [19]:

$$
\text { Total MCC production efficiency }=\mathrm{Ceq}_{\mathrm{MCC}} /\left(\mathrm{Ceq}_{\mathrm{ethanol}}+\mathrm{Ceq}_{\mathrm{SCCs}}\right)_{\text {added }}
$$

where $\mathrm{Ceq}_{\mathrm{MCCs}}$ is the Ceq of the total MCCs produced, which is broadly defined as including $n$-butyrate in this study. Ceqethanol and CeqsCCs represent the Ceq of ethanol and the SCCs, respectively.

To evaluate the specific MCC production, including every individual MCC product, the specific MCC production efficiency was defined as the ratio of Ceq of the specific MCC produced to that of the initial carbon source added, as Equation (2):

$$
\text { Specific MCC production efficiency }=\mathrm{Ceq}_{\text {specific } \mathrm{MCC}} /\left(\mathrm{Ceq}_{\mathrm{ethanol}}+\mathrm{Ceq}_{\mathrm{SCCs}}\right)_{\text {added }}
$$

where $\mathrm{Ceq}_{\text {specific MCC }}$ is the Ceq of the specific MCC produced. The sum of the specific MCC production efficiency is equal to the total MCC production efficiency.

The residual carbon efficiency characterizes the extent to which the substrate was not converted to longer chain products, which is the ratio of Ceq of the total residual substrate to that of the initial carbon source added, as shown in Equation (3):

$$
\text { Residual carbon efficiency }=\left(\mathrm{Ceq}_{\mathrm{ethanol}}+\mathrm{Ceq}_{\mathrm{SCC} s}\right)_{\text {residual }} /\left(\mathrm{Ceq}_{\mathrm{ethanol}}+\mathrm{Ceq}_{\mathrm{SCC}}\right)_{\mathrm{added}} \text {. }
$$

To evaluate the specific organic residual, including every individual VFA substrate, the specific residual organic efficiency was defined as the ratio of Ceq of the specific organic residual to that of the initial carbon source added, as Equation (4):

Specific residual organic efficiency $=\mathrm{Ceq}_{\text {specific residual organic }} /\left(\mathrm{Ceq}_{\mathrm{ethanol}}+\mathrm{Ceq}_{\mathrm{SCCs}}\right)_{\text {added }}$

where $\mathrm{Ceq}_{\text {specific residual organic }}$ is the Ceq of the specific organic residual.

The lost carbon efficiency represents the net loss of the carbon source in the chain elongation system, being calculated as the ratio of Ceq of the lost carbon to that of the initial carbon source added in Equation (5):

Lost carbon efficiency $=1-[($ Ceqethanol + CeqSCCs)residual + CeqMCCs $] /($ Ceqethanol + CeqSCCs)added

\section{Results and Discussion}

\subsection{Effect of the Acetate/Ethanol Ratio on Chain Elongation}

\subsubsection{Performance of Chain Elongation with Different Acetate/Ethanol Ratios}

The concentration variations of substrates and products along the 12 day chain elongation period are shown in Figure 1. The chain elongation with different acetate/ethanol ratios all can be divide into lag phase, rapid reaction phase, and stable phase. However, the duration of these three phases was different for different acetate/ethanol ratios. The lag phase with the acetate/ethanol ratios of 2:1, 1:1, 1:2, and $1: 3$ was $3 \mathrm{~d}$, and was shortened to $2 \mathrm{~d}$ with the decrease of the acetate/ethanol ratio to 1:4. The rapid reaction phase with the acetate/ethanol ratios of 2:1 and 1:1 was the shortest at $1 \mathrm{~d}$, and was prolonged 
to $2 \mathrm{~d}$ with the increase of the acetate/ethanol ratio to 1:2, 1:3, and further increased to $4 \mathrm{~d}$ with the acetate/ethanol ratio of 1:4. It could be speculated that within a certain range, a higher proportion of added ethanol resulted in a shorter lag phase and a longer rapid reaction phase for chain elongation with acetate as the electron acceptor. The higher concentration of ethanol might stimulate the growth of chain elongation bacteria to promote chain elongation and increase the reaction degree. In the lag phase, the substrate concentration varied in scope, in which the ethanol concentration decreased slowly, and the acetate concentration increased slightly. There was almost no MCC produced in this stage, while the microbes adapted to the new environment, synthesized, and stored enough enzymes, energy, and intermediate metabolites for their growth. In the rapid reaction phase, the acetate and ethanol were consumed rapidly, producing a large amount of $n$-butyrate and a small quantity of $n$-caproate through the chain elongation process. During the stable phase, the composition of the system tended to be steady because of substrate deficiency. Furthermore, in the rapid reaction phase, a more rapid increase of $n$-butyrate production was observed than that of $n$-caproate, which may be derived from the rule of adding two carbons at one time and the regulation of acetate elongation to $n$-butyrate and the latter elongation to $n$-caproate [28].
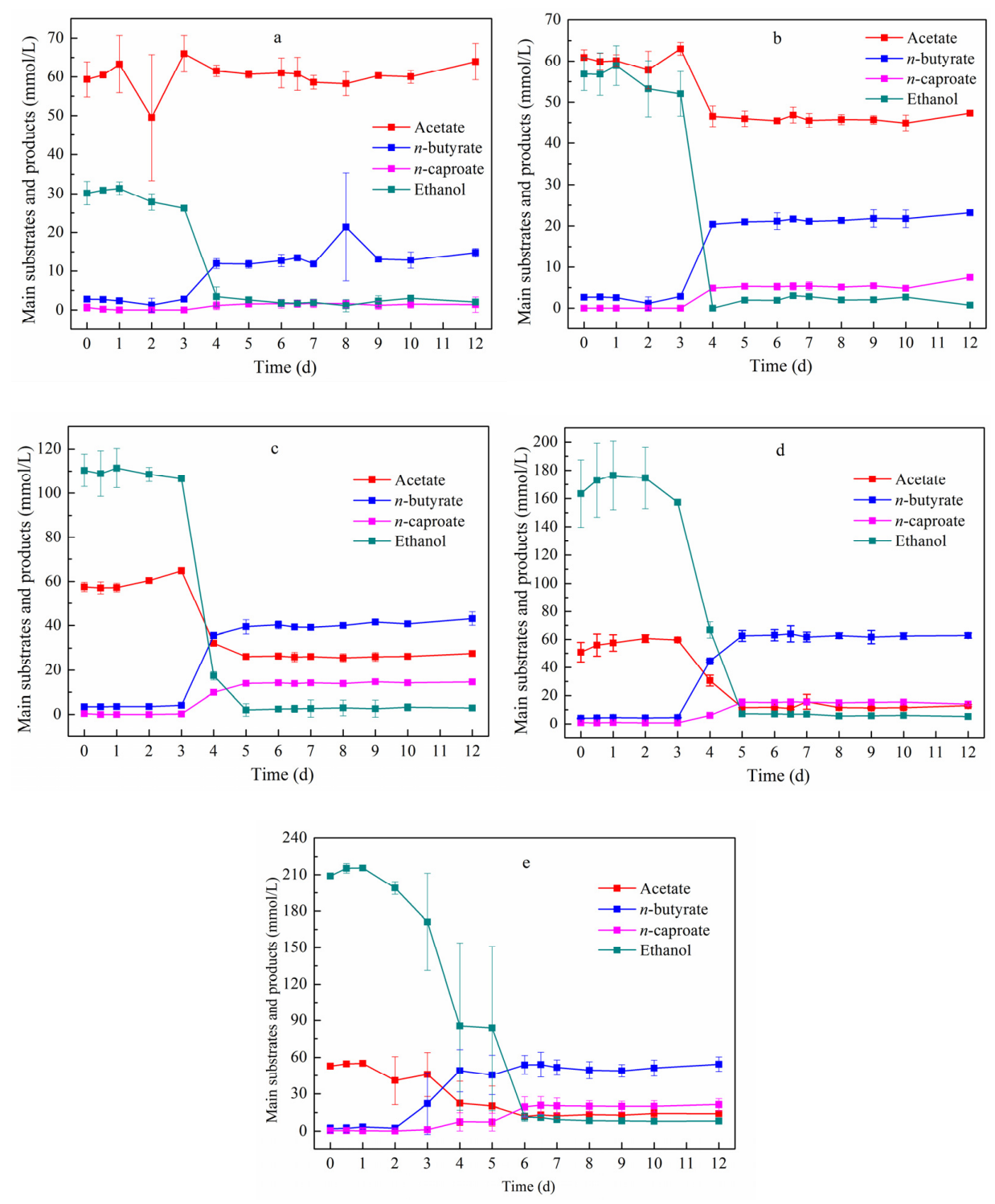

Figure 1. Concentration variation of main substrates and products along chain elongation with acetate/ethanol ratios of 2:1 (a), 1:1 (b), 1:2 (c), 1:3 (d), and 1:4 (e). 


\subsubsection{Comparison of Chain Elongation with Different Acetate/Ethanol Ratios}

We choose the highest reaction degree in the stable phase (day 12, day 12, day 12, day 6.5, and day 12 for acetate/ethanol ratios of 2:1, 1:1,1:2,1:3, and 1:4, respectively) to compare the chain elongation performance with different acetate/ethanol ratios, as shown in Figure 2. It was found that the consumption of ethanol increased with the decrease of acetate/ethanol ratios from 2:1 to 1:4. The consumption of acetate and the total production of fatty acid increased with the decrease of the acetate/ethanol ratio from 2:1 to 1:3, and then decreased slightly from 1:3 to 1:4. Among the MCC products, the largest $n$-butyrate production of $60.03 \mathrm{mmol} / \mathrm{L}$ was achieved with the acetate/ethanol ratio of 1:3, while the largest $n$-caproate production of $20.09 \mathrm{mmol} / \mathrm{L}$ was achieved with the acetate/ethanol ratio of 1:4. The increase of $n$-caproate production with the decrease of acetate/ethanol ratio from 2:1 to 1:4 indicated that a higher acetate/ethanol ratio was more conducive to the conversion of $n$-butyrate to n-caproate, which accorded with the results of other research [29-31], therefore, 1:4 was the optimal acetate/ethanol ratio for acetate chain elongation. However, the chain elongation with acetate as the electron acceptor did not have a high conversion efficiency to $n$-caproate. The accumulation of $n$-butyrate might be attributed to the low partial pressure of hydrogen in the chain elongation system [17].


Figure 2. Substrate consumption (negative values) and product formation (positive values) (a), and fatty acid product distribution $(\mathbf{b})$ with acetate as the electron acceptor at the maximum reaction degree.

\subsection{Effect of the Propionate/Ethanol Ratio on Chain Elongation}

\subsubsection{Performance of Chain Elongation with Different Propionate/Ethanol Ratios}

The concentration variations of substrates and products along the 12 days chain elongation period are shown in Figure 3. The chain elongation with propionate as the electron acceptor also presented the lag phase, rapid reaction phase, and stable phase. The lag phase with the propionate/ethanol ratio of $2: 1$ was $7 \mathrm{~d}$, and was shortened to $6 \mathrm{~d}$ with the propionate/ethanol ratio of 1:1, and was the shortest with the propionate/ethanol ratios of 1:2, 1:3, and 1:4. The rapid reaction phase in all experiment groups was about $2 \mathrm{~d}$. A higher proportion of added ethanol resulted in a shorter lag phase with propionate as electron acceptor. In the lag phase, there was an obvious decrease of ethanol and propionate concentrations with continuous production of acetate. Similar to the "production of hydrogen and acetate" in the anaerobic digestion process, ethanol and propionate were converted into acetate, carbon dioxide, and hydrogen by acetogens. This process is known as substrate oxidation and was the major reason for the substrate conversion almost without MCC generation in the lag phase [32]. The rapid reaction phase of propionate chain elongation could be further divided into the propionate elongation phase and acetate elongation phase, and these two reaction phases overlapped each other. In the propionate elongation phase, propionate and ethanol were consumed rapidly to produc $n$-valerate; in the acetate elongation phase, similar to the chain elongation with acetate as the electron acceptor, acetate and ethanol were consumed as substrates to produce $n$-butyrate and $n$-caproate simultaneously (Figure 3). Therefore, the main products with propionate as the electron 
acceptor were not only $n$-valerate, but also $n$-butyrate and $n$-caproate, which accorded with the results from Bornstein and Barker [29]. Obviously, the acetate produced from propionate oxidation promoted the even-carbon chain elongation.
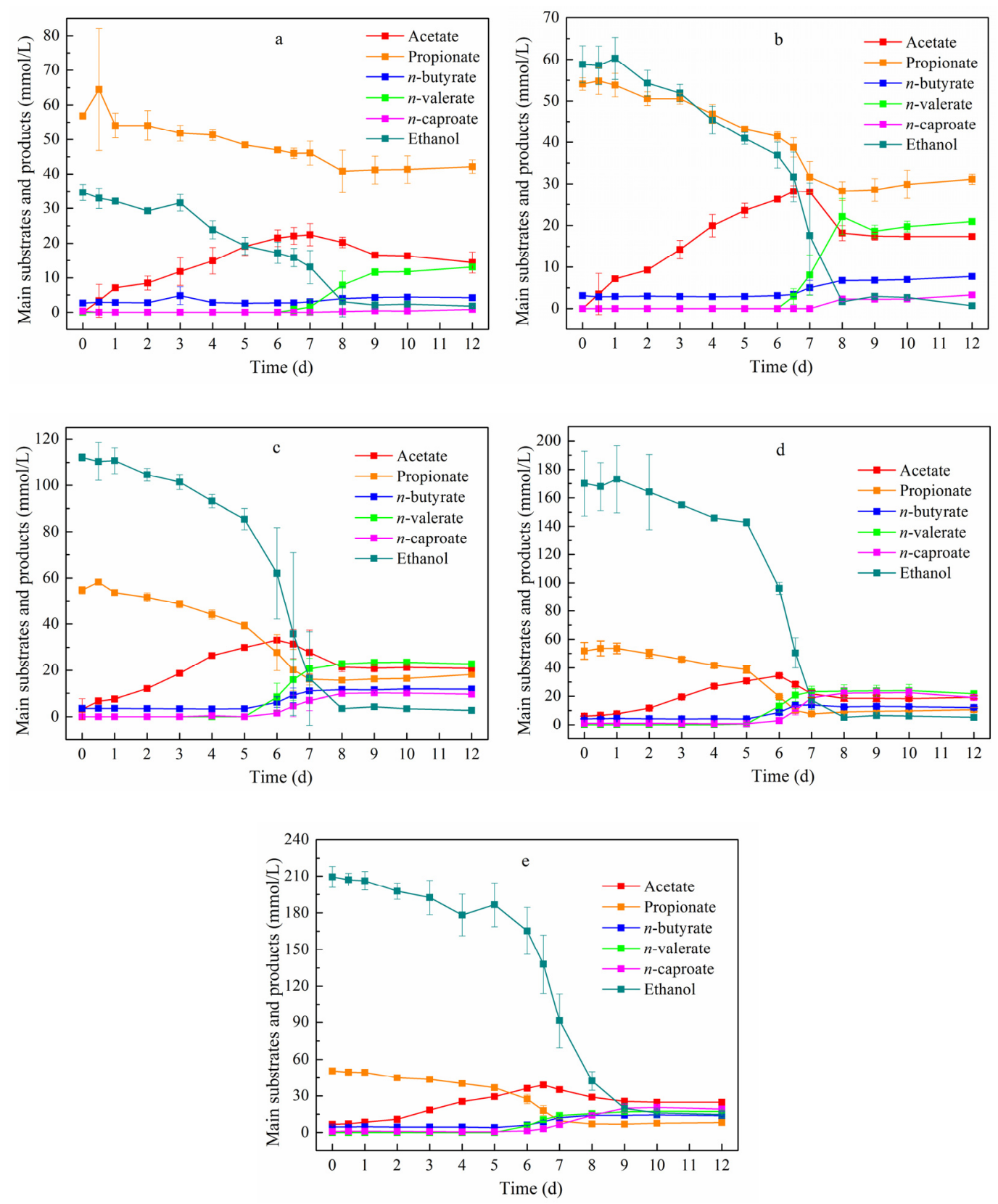

Figure 3. Concentration variation of main substrates and products along chain elongation with propionate/ethanol ratios of 2:1 (a), 1:1 (b), 1:2 (c), 1:3 (d), and 1:4 (e).

\subsubsection{Comparison of Chain Elongation with Different Propionate/Ethanol Ratios}

The highest reaction degree in the stable phase (day 12 , day 12 , day 10 , day 10 , and day 10 for propionate/ethanol ratios of 2:1, 1:1, 1:2, 1:3, and 1:4, respectively) was chosen to compare the chain elongation performance with different propionate/ethanol ratios, as shown in Figure 4. Similar with the chain elongation of acetate, the consumption of ethanol increased with the decrease of the propionate/ethanol ratio from 2:1 to 1:4. The consumption of propionate and the total production of fatty acid increased with the decrease of the propionate/ethanol ratio from 2:1 to 1:3, and then decreased slightly from 1:3 to 1:4. The production of $n$-valerate (about $23 \mathrm{mmol} / \mathrm{L}$ ) was similar with 
the propionate/ethanol ratios of 1:2 and 1:3; while the production of the target product $n$-caproate (about $20 \mathrm{mmol} / \mathrm{L}$ ) was similar with the propionate/ethanol ratios of 1:3 and 1:4. Both the largest production of the main products $n$-valerate $(23.97 \mathrm{mmol} / \mathrm{L})$ and $n$-caproate $(21.44 \mathrm{mmol} / \mathrm{L})$ were achieved with the propionate/ethanol ratio of 1:3. What is more, the chain elongation with propionate as the electron acceptor realized the effective conversion from $n$-butyrate to $n$-caproate with the propionate/ethanol ratio of 1:3. The best chain elongation performance suggested that 1:3 was the optimal propionate/ethanol ratio for chain elongation with propionate as the electron acceptor. In addition, as shown in Figure $4 \mathrm{~b}$, there was a small amount of $n$-heptanoate produced $(1.61 \mathrm{mmol} / \mathrm{L}$ for the propionate/ethanol ratio of 1:3).
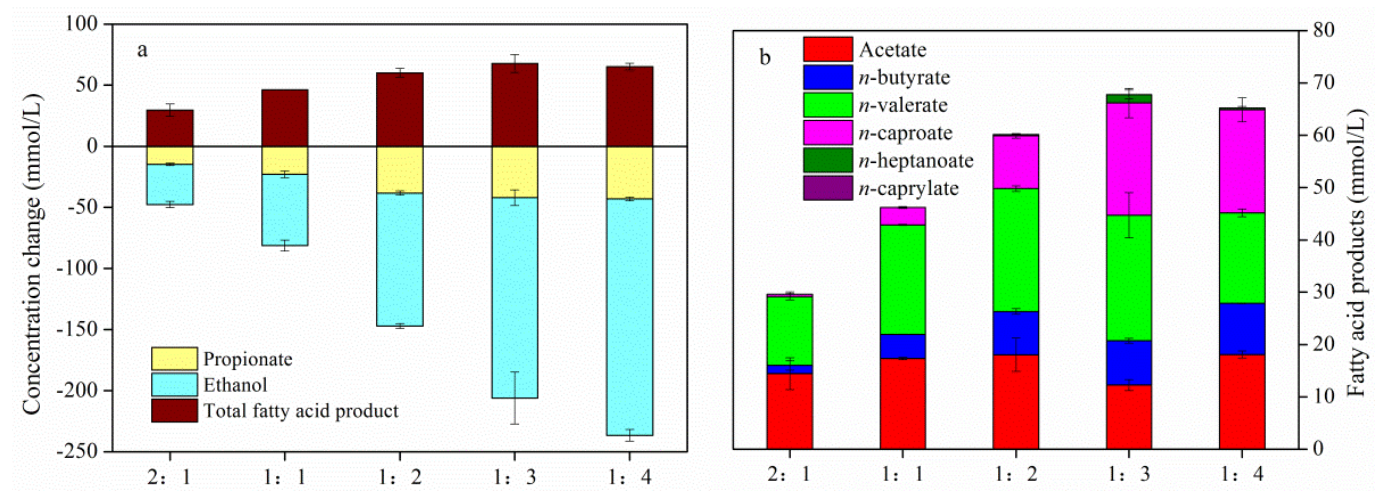

Figure 4. Substrate consumption (negative values) and product formation (positive values) (a) and fatty acid product distribution (b) with propionate as the electron acceptor under the maximum reaction degree.

\subsection{Effect of the n-Butyrate/Ethanol Ratio on Chain Elongation}

\subsubsection{Performance of Chain Elongation with Different $n$-Butyrate/Ethanol Ratios}

As shown in Figure 5, the chain elongation with $n$-butyrate as the electron acceptor also presented a lag phase, rapid reaction phase, and stable phase. The lag phase with the $n$-butyrate/ethanol ratios of 2:1 and 1:1 was $6 \mathrm{~d}$, and was shortened to $4 \mathrm{~d}$ with the $n$-butyrate/ethanol ratios of 1:2, 1:3, and 1:4. The rapid reaction phase with all $n$-butyrate/ethanol ratios was about $2 \mathrm{~d}$. A higher proportion of added ethanol corresponded to a shorter lag phase with $n$-butyrate as the electron acceptor. In the lag phase, the acetate was continuously generated, while the concentration of ethanol and $n$-butyrate decreased, almost without any chain elongation (Figure 5). This phenomenon is similar to that with propionate as the electron acceptor, where both ethanol oxidation and propionate or $n$-butyrate oxidation occurred. In the rapid reaction phase, the $n$-butyrate and ethanol were rapidly consumed to produce a large amount of $n$-caproate through chain elongation. It is worth noting that the concentration of acetate generated in the lag phase slightly decreased from day 5 to day 6 with the $n$-butyrate/ethanol ratios of $1: 2$ and 1:3, indicating that the chain elongation of acetate also occurred in these reaction systems, which could be named as acetoclastic caprogenesis. It is obvious that the acetate elongation to $n$-butyrate might be driven by the $n$-butyrate reduction due to the $n$-butyrate elongation to $n$-caproate, which indicated a better chain elongation effect with the $n$-butyrate/ethanol ratios of 1:2 and 1:3. 

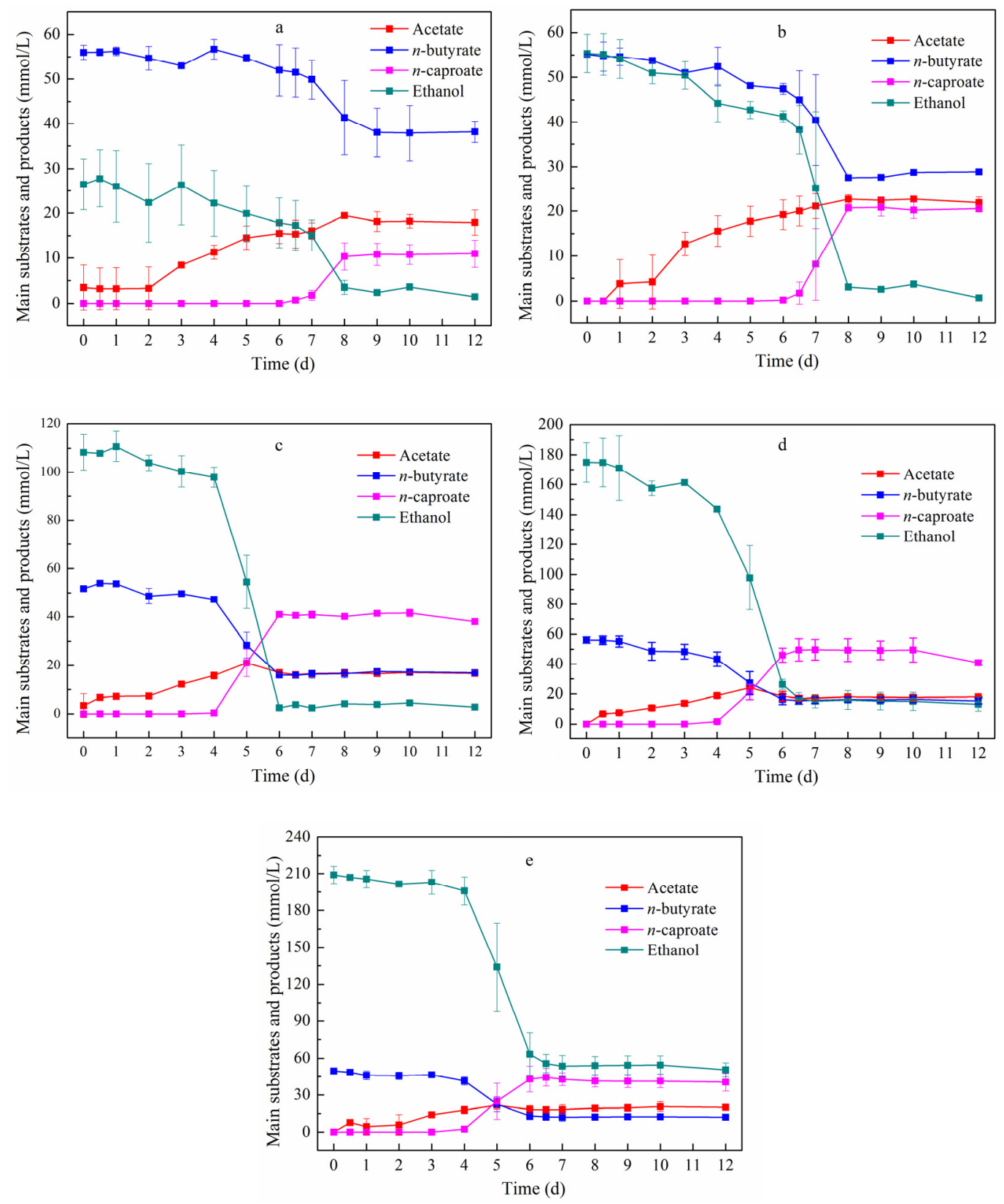

Figure 5. Concentration variation of main substrates and products along chain elongation with n-butyrate/ethanol ratios of 2:1 (a), 1:1 (b), 1:2 (c), 1:3 (d), and 1:4 (e).

\subsubsection{Comparison of Chain Elongation with Different $n$-Butyrate/Ethanol Ratios}

The highest reaction degree in the stable phase (day 12 , day 9 , day 10 , day 8 , and day 9 for $n$-butyrate/ethanol ratios of 2:1, 1:1,1:2, 1:3, and 1:4, respectively) with different $n$-butyrate/ethanol ratios is shown in Figure 6. Different from the chain elongation of acetate and propionate, both the consumption of ethanol and $n$-butyrate increased with the decrease of $n$-butyrate/ethanol ratios from 2:1 to 1:3, and then decreased slightly from 1:3 to 1:4. The same variation trend was also observed in the total production of fatty acids and $n$-caproate. The largest production of targeted $n$-caproate of $49.36 \mathrm{mmol} / \mathrm{L}$ was achieved with the $n$-butyrate/ethanol ratio of 1:3. Compared to 1:3, the similar $n$-caproate production of $41.54 \mathrm{mmol} / \mathrm{L}$ was achieved with the $n$-butyrate/ethanol ratio of 1:2 but consumed less ethanol, indicating that a higher $n$-caproate production efficiency might be achieved with the ratio of 1:2. What is more, the chain elongation with $n$-butyrate as the electron acceptor realized the effective conversion from $n$-butyrate to $n$-caproate with the $n$-butyrate/ethanol ratios of 1:2, 
$1: 3$, and 1:4. In addition, as shown in Figure $6 \mathbf{b}$, there was a small amount of $n$-caprylate production (2.35 and $4.75 \mathrm{mmol} / \mathrm{L}$ for the propionate/ethanol ratios of 1:3 and 1:4, respectively).
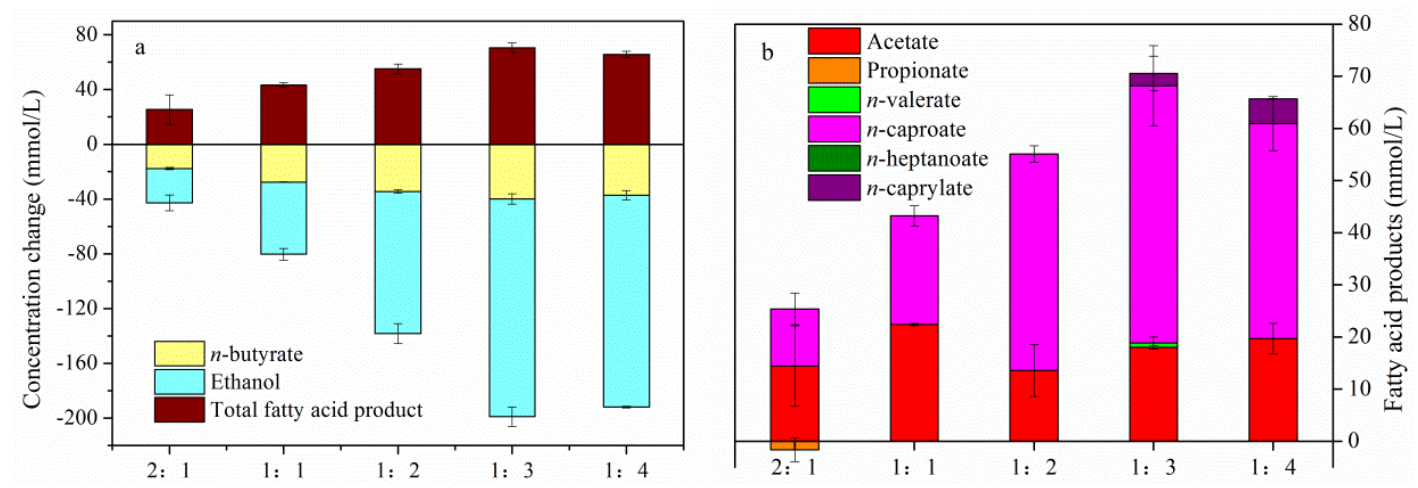

Figure 6. Substrate consumption (negative values) and product formation (positive values) (a) and fatty acid product distribution (b) with $n$-butyrate as the electron acceptor under the maximum reaction degree.

\subsection{Evaluation of the Carbon Balance in Chain Elongation Systems}

\subsubsection{Chain Elongation Carbon}

In this study, total MCC production efficiency was used to evaluate the performance of overall chain elongation. Meanwhile, the specific MCC production efficiency was applied to evaluate the conversion to specific MCC products, as shown in Figure 7. In the chain elongation with acetate as the electron acceptor, the highest total MCC production efficiency and $n$-butyate production efficiency were achieved with the acetate/ethanol ratio of 1:3, while the $n$-caproate production efficiency with the acetate/ethanol ratios of $1: 2$ and $1: 4$ was similarly high with $24.99 \%$ and $24.08 \%$, respectively. In the chain elongation with propionate as the electron acceptor, the highest $n$-valerate production efficiency was achieved with the acetate/ethanol ratio of 1:1, while both the highest total MCC production efficiency and $n$-caproate production efficiency were achieved with the propionate/ethanol ratio of 1:3, which accorded with the best performance of chain elongation. In the chain elongation with $n$-butyrate as the electron acceptor, the $n$-caproate production efficiency with the $n$-butyrate/ethanol of 1:2 was the highest with $57.96 \%$, which is equal to the total MCC production efficiency. Considering the relatively higher $n$-caproate production and the highest $n$-caproate production efficiency, 1:2 was the optimal $n$-butyrate/ethanol ratio. This conclusion confirmed the proposal by Ge et al. [24]. Overall, the total MCC production efficiency increased first with the increase of initial ethanol concentration; after reaching the peak value, it was reduced with the subsequent increase of the initial ethanol concentration. Therefore, the extra ethanol addition was detrimental to the carbon conversion efficiency for the total MCC production. Furthermore, it can be observed in Figure 7 that both the variation of $n$-butyrate production efficiency with acetate as the electron acceptor and the variation of $n$-caproate production efficiency with propionate and $n$-butyrate as the electron acceptor were consistent with the total MCC production efficiency. Therefore, the extra ethanol addition was also detrimental to the main MCC production. 


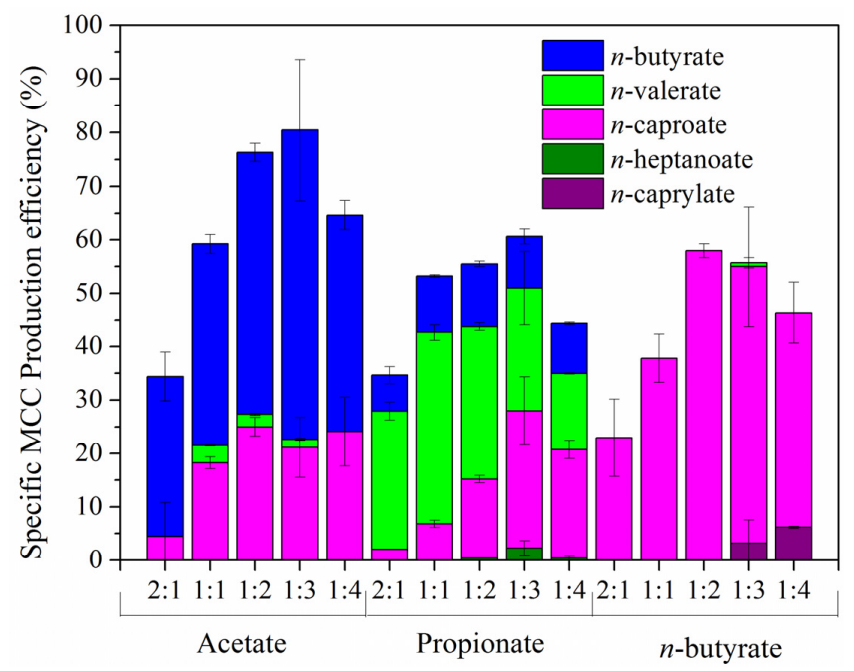

Figure 7. Specific medium chain carboxylate (MCC) production efficiency at different acid/ethanol ratios with acetate, propionate, and $n$-butyrate as the electron acceptor, respectively (Total MCC production efficiency is equal to the sum of each specific MCC production efficiency).

\subsubsection{Residual and Lost Carbon}

Less than $100 \%$ of the carbon took part in the chain elongation, moreover, some carbons were consumed by competition processes. To further clarify the reasons for the reduction of the total MCC production efficiency with the extra ethanol addition, the carbon was divided into three parts: total MCC production efficiency, residual carbon efficiency, and lost carbon efficiency (Figure 8). The lost carbon was turned into carbon dioxide via substrate oxidation and to methane via subsequent hydrotrophic methanogenesis. The lost carbon efficiency increased with the increase of initial ethanol concentration, which indicated that higher ethanol addition promoted the ethanol oxidation process. The loss of ethanol decreased the MCC production efficiency because of the insufficient electron donor. This is the reason why the optimal acetate/ethanol ratio of 1:4 under the open culture system is smaller than that with pure bacteria culture [18], because it is necessary to ensure sufficient ethanol utilization for chain elongation.

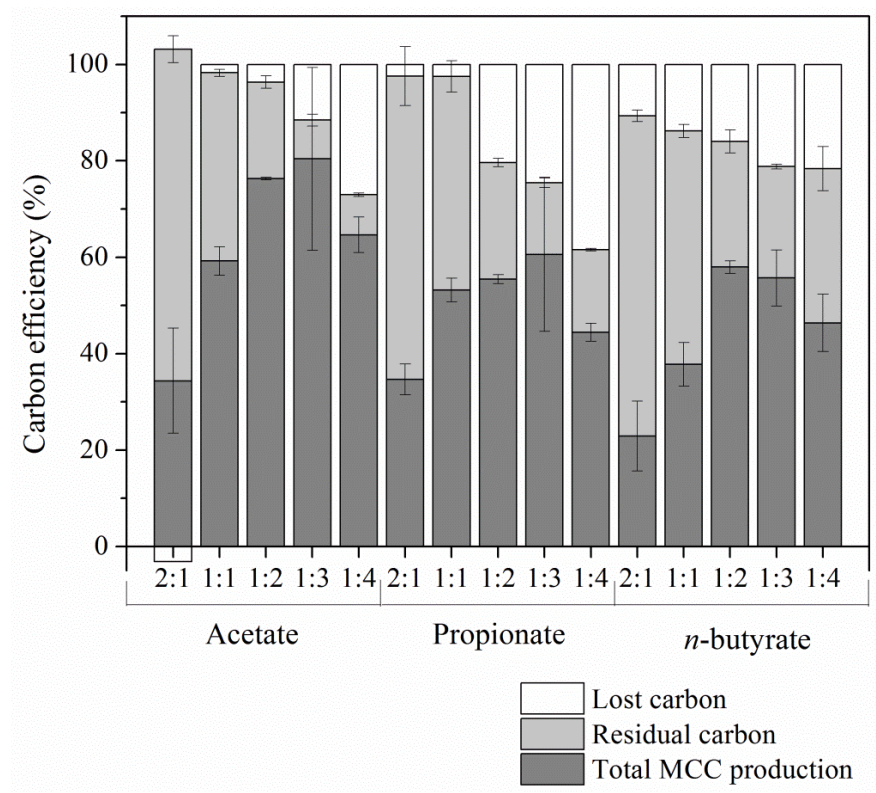

Figure 8. Lost carbon efficiency, residual carbon efficiency, and total MCC production efficiency at different acid/ethanol ratios with acetate, propionate, and $n$-butyrate as the electron acceptor. 
The residual carbon efficiency characterized the degree of chain elongation from another aspect, because the variation of total production efficiency went against with the variation of the residual carbon efficiency. The relatively lower residual carbon efficiencies of $8.03 \%, 14.83 \%$, and $26.07 \%$ with an acetate/ethanol ratio of 1:3, propionate/ethanol ratio of 1:3, and $n$-butyrate/ethanol ratio of 1:2 manifested the better chain elongation realization with sufficient ethanol addition under the corresponding optimal acid/ethanol ratio. The highest MCC production efficiencies with different electron acceptors were consistent with less carbon loss and higher chain elongation degree.

To further clarify the chain elongation degree, we divided the residual carbon efficiency into several parts as the compositions of residual organics. As shown in Figure 9, the residual electron acceptor (acetate, propionate, and $n$-butyrate) efficiency basically decreased with the increase of initial ethanol concentration, and the residual electron donor (ethanol) efficiency increased with the increase of initial ethanol concentration with acetate, propionate, and $n$-butyrate as the electron acceptor. It seems that the absence of ethanol resulted in the residual electron acceptor with a higher acid/ethanol ratio than the optimal one, and the extra addition of ethanol resulted in the residual electron donor. Therefore, with the optimal acid/ethanol ratio, the substrates were maximally utilized for chain elongation. What is more, the residual ethanol efficiency from the extra ethanol increased from acetate to $n$-butyrate as the electron acceptor (Figure 9). From this phenomenon, it can be speculated that the longer the carbon chain length of the electron acceptor, the less carbon there is from ethanol for $n$-caproate production, thus, the more ethanol carbon there is as residual. This finding powerfully explained why the highest $n$-caproate production efficiency was achieved with an acid/ethanol ratio increase with acetate, propionate, and $n$-butyrate as the electron acceptor (the optimal acid/ethanol ratios of 1:4, 1:3, and 1:2, respectively). In addition, a small amount of propionate production in the even-carbon chain elongation with acetate as the electron acceptor might be derived from the degradation of the inocula and yeast extraction, and the total carbon efficiency higher than $100 \%$ with the acetate/ethanol ratio of 2:1 (Figure 8) might be due to the small amount of VFAs produced from the biodegradation of macromolecular organics [19].

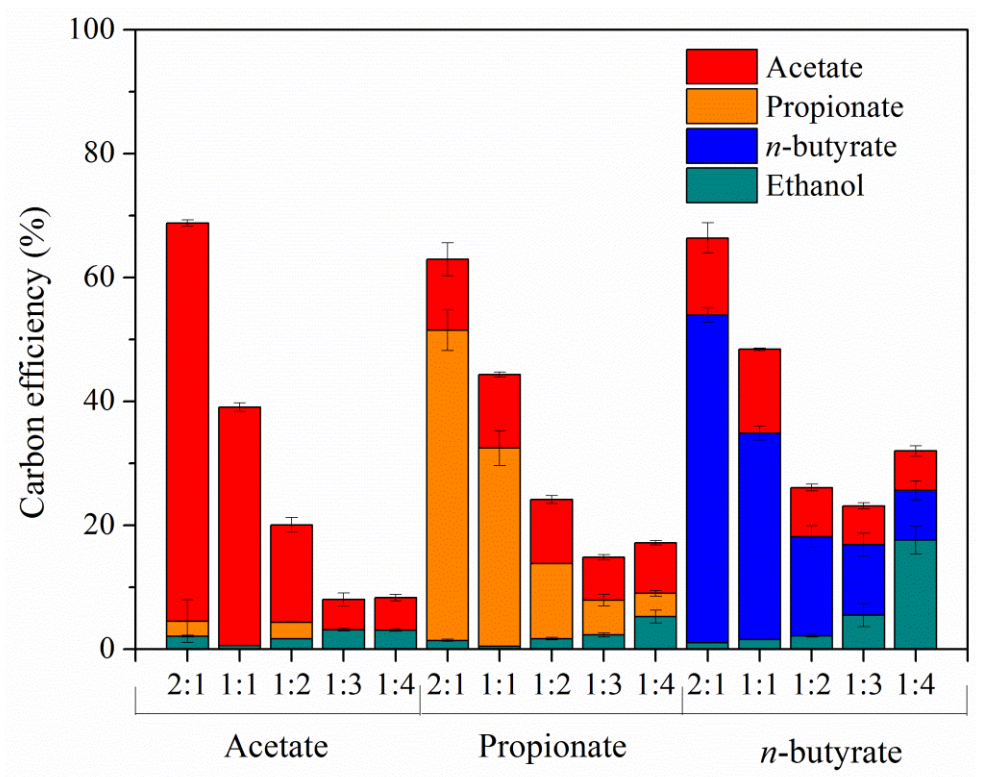

Figure 9. Specific residual organic efficiency at different acid/ethanol ratios with acetate, propionate, and $n$-butyrate as the electron acceptor (Residual carbon efficiency equal to the sum of each residual organic efficiency in the chain elongation system).

\subsection{Microbial Community Analysis}

The microbial samples were collected almost at the end of the rapid reaction phase. For the chain elongation with acetate as the electron acceptor, the sample collection was on the day 4, day 4 , 
day 5, day 5, and day 6.5 with the acetate/ethanol ratios of 2:1, 1:1, 1:2, 1:3, and 1:4, respectively; with propionate as the electron acceptor, collection was on the day 9 , day 8 , day 8 , day 8 , and day 9 , respectively; and with $n$-butyrate as the electron acceptor, collection was on the day 8 , day 8 , day 6 , day 6.5, and day 6, respectively. Figure 10 shows the phylogenetic classification of microbes at phylum (Figure 10a) and genus (Figure 10b) level. Generally, the bacterial communities were composed of eight phyla, higher than $96.7 \%$ of the total sequences. Among them, three phyla Firmicutes, Bacteroidetes, and Proteobacteria mainly dominated in the chain elongation systems with acetate $(76.25-82.22 \%)$, propionate (75.6-84.4\%), and $n$-butyrate (74.7-85.3\%) as the electron acceptor. The total relative abundances of these three phyla were quite similar with different electron acceptors, while they varied obviously with different acid/ethanol ratios, for example, $61.6-64.2 \%$ and $67.2-70.1 \%$ with the acid/ethanol ratios of 2:1 and 1:1, respectively (Figure 10a). The population distribution at the phylum level with different acid/ethanol ratios indicated that the stimulation of ethanol had a great influence on the transformation of the microbial community.

Genera with relative abundances higher than $2 \%$ in each sample (a total of 16 genera) are displayed in Figure 10b, and five recognized genera, Clostridium_sensu_stricto_12 (19.9-60.6\%), Sporanaerobacter (3.9-8.1\%), Proteiniphilum (1.9-8.9\%), Lachnospiraceae_NK3A20_group (2.2-5.5\%), and Syntrophobacter $(1.8-4.2 \%)$ predominated in all fifteen chain elongation systems. Clostridium_sensu_stricto_12 was obviously enriched in all systems, which accounted for most of the bacteria with an abundance of $20.7-53.1 \%, 19.9-52.7 \%$, and $25.2-60.6 \%$, when the acetate, propionate, $n$-butyrate were used as the electron acceptor, respectively. Clostridium_sensu_stricto_12 was reported to have the ability of chain elongation to $n$-butyrate and $n$-caproate [33], and the well-known $n$-caproate producing species C. kluyveri belongs to Clostridium_sensu_stricto_12 [34]. Therefore, the $n$-caproate production from the chain elongation reaction was derived from the enrichment of Clostridium_sensu_stricto_12. The research by Yang et al. [34] reported that ethanol is an important factor to this type of $n$-caproate producer. To further clarify the effect of ethanol on Clostridium_sensu_stricto_12, the relative abundance of this genus in different systems was compared. As shown in Figure 10b, with acetate as the electron acceptor, the relative abundance of Clostridium_sensu_stricto_12 basically increased with the decrease of acetate/ethanol ratios from 2:1 to 1:4, and reached the highest of $53.1 \%$ at the acetate/ethanol ratio of 1:4 (Figure 10b). With both propionate and $n$-butyrate as the electron acceptor, the relative abundance of Clostridium_sensu_stricto_12 first increased to the peak and then decreased with the decrease of acid/ethanol ratios from $2: 1$ to $1: 4$, and the peaks were $52.7 \%$ and $60.6 \%$ at the acid/ethanol ratio of $1: 3$ and 1:2 with propionate and $n$-butyrate as the electron acceptor, respectively. The optimal acid/ethanol ratio for the highest relative abundance gradually increased with acetate and $n$-butyrate as the electron acceptors $(1: 4,1: 3$, and 1:2); this regulation is the same as the variation regulation of the optimal acid/ethanol ratio for the $n$-caproate production, which indicated that it is the optimal acid/ethanol ratio with the higher $n$-caproate production rather than the higher ethanol addition that favored a higher relative abundance of $n$-caproate producers. One reasonable explanation for this conclusion is that the longer the carbon chain of MCCs, the stronger reducibility the chain elongation products, and the more ATP is released, which is advantageous for chain elongating bacteria $[8,35]$. It is worth noting that this conclusion was obtained with a low ethanol concentration $(<300 \mathrm{mmol} / \mathrm{L})$ and no inhibition to microbes [19].

Furthermore, there was an obvious decrease of genus Clostridium_sensu_stricto_1 when the acid/ethanol ratio varied from 2:1 to 1:4 with all three electron acceptors (Figure 10b). It has been reported that the chain elongation intermediate, $n$-butyrate, is a genus-specific product from macromolecular organic fermentation of Clostridium_sensu_stricto_1 [34]. The lack of ethanol may require more $n$-butyrate as the electron acceptor to fulfill the need of the $n$-caproate production, thus, some $n$-butyrate was directly produced with such bacteria. The enrichment of proteolytic bacteria Proteiniphilum might be attributed to the addition of $1 \mathrm{~g} / \mathrm{L}$ yeast extraction, which could use proteins and amino acids as a fermentative substrate to produce carbon dioxide, hydrogen, and SCCs [36]. The enrichment of Lachnospiraceae_NK3A20_group, responsible for the dehydrogenation of fatty acids [37], may also be 
connected with the macromolecule organic degradation and further conversion into SCCs, such as acetate and $n$-butyrate, especially for the acetate production in Figure 2a.


Figure 10. Bacterial abundance in chain elongation systems on phylum level (a) and on genus level (b).

There were also some acetogenic genera, such as Petrimonas [38] and Syntrophobacter, the relative abundance of which was relatively lower with the optimal acid/ethanol ratio (Figure 10b). Syntrophobacter, as a syntrophism bacteria, can form a syntrophism system only with hydrotrophic methanogens to conduct the oxidation of ethanol, propionate, or $n$-butyrate to acetate and hydrogen $[39,40]$. The relative abundance of substrate oxidation bacteria Syntrophobacter was almost inversely correlated with the $n$-caproate production efficiency (also the relative abundance of Clostridium_sensu_stricto_12) with different acid/ethanol ratios. It is obvious that the extra ethanol and residual carbon boosted the competition bacteria, then decreased the proportion of chain elongation bacteria, which could be another reasonable explanation for the higher relative abundance of $n$-caproate production under the optimal acid/ethanol ratio.

\section{Conclusions}

The better performance of MCCs, especially the $n$-caproate production, supported the optimal acid/ethanol ratios of 1:4, 1:3, and 1:2 with acetate, propionate, and $n$-butyrate as the electron acceptor, 
respectively. There are similar regulations with acetate, propionate, and $n$-butyrate as the electron acceptor: the higher concentration of ethanol stimulated the growth of chain elongation bacteria to promote chain elongation and increase the reaction degree. The highest MCC production efficiency with different electron acceptors were consistent with less carbon loss and higher chain elongation degree. In addition, with the optimal acid/ethanol ratio, the substrate was maximally utilized for chain elongation. The microbial community showed that a higher $n$-caproate production was achieved with the optimal acid/ethanol ratio rather than the higher ethanol addition, which was beneficial to a higher relative abundance of $n$-caproate producers.

Author Contributions: Conceptualization, P.Z. and S.B.; investigation, S.B., Q.Z., and Y.Z.; software, S.W.; validation, F.L. and Y.W.; formal analysis, S.B., X.T., and M.N.; writing-original draft preparation, S.B. and Q.W.; writing-review and editing, S.B. and P.Z.

Acknowledgments: This research was funded by Fundamental Research Funds for The Central Universities, Grant Number 2017cgp017 And National Natural Science Foundation of China, grant number 51578068.

Conflicts of Interest: The authors declare no conflict of interest. The funders had no role in the design of the study; in the collection, analyses, or interpretation of data; in the writing of the manuscript, or in the decision to publish the results.

\section{References}

1. Nabi, M.; Zhang, G.; Zhang, P.; Tao, X.; Wang, S.; Ye, J.; Zhang, Q.; Zubair, M.; Bao, S.; Wu, Y. Contribution of solid and liquid fractions of sewage sludge pretreated by high pressure homogenization to biogas production. Bioresour. Technol. 2019, 286, 121378. [CrossRef] [PubMed]

2. Wang, S.; Li, F.; Wu, D.; Zhang, P.; Wang, H.; Tao, X.; Ye, J.; Nabi, M. Enzyme pretreatment enhancing biogas yield from corn stover: Feasibility, optimization, and mechanism analysis. J. Agric. Food Chem. 2018, 66, 10026-10032. [CrossRef] [PubMed]

3. Ye, J.; Hu, A.; Cheng, X.; Lin, W.; Liu, X.; Zhou, S.; He, Z. Response of enhanced sludge methanogenesis by red mud to temperature: Spectroscopic and electrochemical elucidation of endogenous redox mediators. Water Res. 2018, 143, 240-249. [CrossRef]

4. Ye, J.; Hu, A.; Ren, G.; Chen, M.; Tang, J.; Zhang, P.; Zhou, S.; He, Z. Enhancing sludge methanogenesis with improved redox activity of extracellular polymeric substances by hematite in red mud. Water Res. 2018, 134, 54-62. [CrossRef] [PubMed]

5. Zhang, S.; Zhang, P.; Zhang, G.; Fan, J.; Zhang, Y. Enhancement of anaerobic sludge digestion by high-pressure homogenization. Bioresour. Technol. 2012, 118, 496-501. [CrossRef] [PubMed]

6. Zaks, D.P.; Winchester, N.; Kucharik, C.J.; Barford, C.C.; Paltsev, S.; Reilly, J.M. Contribution of anaerobic digesters to emissions mitigation and electricity generation under US climate policy. Environ. Sci. Technol. 2011, 45, 6735-6742. [CrossRef] [PubMed]

7. Agler, M.T.; Wrenn, B.A.; Zinder, S.H.; Angenent, L.T. Waste to bioproduct conversion with undefined mixed cultures: The carboxylate platform. Trends Biotechnol. 2011, 29, 70-78. [CrossRef]

8. Angenent, L.T.; Richter, H.; Buckel, W.; Spirito, C.M.; Steinbusch, K.J.; Plugge, C.M.; Strik, D.P.; Grootscholten, T.I.; Buisman, C.J.; Hamelers, H.V. Chain elongation with reactor microbiomes: Open-culture biotechnology to produce biochemicals. Environ. Sci. Technol. 2016, 50, 2796-2810. [CrossRef] [PubMed]

9. Levy, P.; Sanderson, J.; Kispert, R.; Wise, D. Biorefining of biomass to liquid fuels and organic chemicals. Enzym. Microb. Technol. 1981, 3, 207-215. [CrossRef]

10. Witholt, B.; Kessler, B. Perspectives of medium chain length poly (hydroxyalkanoates), a versatile set of bacterial bioplastics. Curr. Opin. Biotechnol. 1999, 10, 279-285. [CrossRef]

11. Spirito, C.M.; Richter, H.; Rabaey, K.; Stams, A.J.; Angenent, L.T. Chain elongation in anaerobic reactor microbiomes to recover resources from waste. Curr. Opin. Biotechnol. 2014, 27, 115-122. [CrossRef] [PubMed]

12. de Araújo Cavalcante, W.; Leitão, R.C.; Gehring, T.A.; Angenent, L.T.; Santaella, S.T. Anaerobic fermentation for n-caproic acid production: A review. Process Biochem. 2017, 54, 106-119. [CrossRef]

13. Leng, L.; Yang, P.; Mao, Y.; Wu, Z.; Zhang, T.; Lee, P.-H. Thermodynamic and physiological study of caproate and 1,3-propanediol co-production through glycerol fermentation and fatty acids chain elongation. Water Res. 2017, 114, 200-209. [CrossRef] 
14. Wu, Q.; Guo, W.; Bao, X.; Meng, X.; Yin, R.; Du, J.; Zheng, H.; Feng, X.; Luo, H.; Ren, N. Upgrading liquor-making wastewater into medium chain fatty acid: Insights into co-electron donors, key microflora, and energy harvest. Water Res. 2018, 145, 650-659. [CrossRef] [PubMed]

15. Schoberth, S.; Gottschalk, G. Considerations on the energy metabolism of Clostridium kluyveri. Arch. Für Mikrobiol. 1969, 65, 318-328. [CrossRef]

16. Thauer, R.K.; Jungermann, K.; Henninger, H.; Wenning, J.; Decker, K. The energy metabolism of Clostridium kluyveri. Eur. J. Biochem. 1968, 4, 173-180. [CrossRef]

17. Steinbusch, K.J.; Hamelers, H.V.; Plugge, C.M.; Buisman, C.J. Biological formation of caproate and caprylate from acetate: Fuel and chemical production from low grade biomass. Energy Environ. Sci. 2011, 4, $216-224$. [CrossRef]

18. Yin, Y.; Zhang, Y.; Karakashev, D.B.; Wang, J.; Angelidaki, I. Biological caproate production by Clostridium kluyveri from ethanol and acetate as carbon sources. Bioresour. Technol. 2017, 241, 638-644. [CrossRef]

19. Liu, Y.; Lü, F.; Shao, L.; He, P. Alcohol-to-acid ratio and substrate concentration affect product structure in chain elongation reactions initiated by unacclimatized inoculum. Bioresour. Technol. 2016, 218, 1140-1150. [CrossRef] [PubMed]

20. Fang, W.; Zhang, P.; Zhang, X.; Zhu, X.; van Lier, J.B.; Spanjers, H. White rot fungi pretreatment to advance volatile fatty acid production from solid-state fermentation of solid digestate: Efficiency and mechanisms. Energy 2018, 162, 534-541. [CrossRef]

21. Li, G.; Zhang, P.; Wang, Y.; Sheng, Y.; Lv, X.; Yin, J. Enhancing biological denitrification with adding sludge liquor of hydrolytic acidification pretreated by high-pressure homogenization. Int. Biodeterior. Biodegrad. 2016, 113, 222-227. [CrossRef]

22. Wang, S.; Tao, X.; Zhang, G.; Zhang, P.; Wang, H.; Ye, J.; Li, F.; Zhang, Q.; Nabi, M. Benefit of solid-liquid separation on volatile fatty acid production from grass clipping with ultrasound-calcium hydroxide pretreatment. Bioresour. Technol. 2019, 274, 97-104. [CrossRef]

23. Coma, M.; Vilchez-Vargas, R.; Roume, H.; Jauregui, R.; Pieper, D.H.; Rabaey, K. Product diversity linked to substrate usage in chain elongation by mixed-culture fermentation. Environ. Sci. Technol. 2016, 50, 6467-6476. [CrossRef]

24. Ge, S.; Usack, J.G.; Spirito, C.M.; Angenent, L.T. Long-term n-caproic acid production from yeast-fermentation beer in an anaerobic bioreactor with continuous product extraction. Environ. Sci. Technol. 2015, 49, 8012-8021. [CrossRef]

25. Grootscholten, T.; Steinbusch, K.; Hamelers, H.; Buisman, C. Chain elongation of acetate and ethanol in an upflow anaerobic filter for high rate MCFA production. Bioresour. Technol. 2013, 135, 440-445. [CrossRef] [PubMed]

26. Zinder, S.H.; Anguish, T.; Cardwell, S.C. Selective inhibition by 2-bromoethanesulfonate of methanogenesis from acetate in a thermophilic anaerobic digestor. Appl. Environ. Microbiol. 1984, 47, 1343-1345.

27. Wang, S.; Zhang, G.; Zhang, P.; Ma, X.; Li, F.; Zhang, H.; Tao, X.; Ye, J.; Nabi, M. Rumen fluid fermentation for enhancement of hydrolysis and acidification of grass clipping. J. Environ. Manag. 2018, 220, 142-148. [CrossRef] [PubMed]

28. Barker, H.; Kamen, M.; Bornstein, B. The synthesis of butyric and caproic acids from ethanol and acetic acid by Clostridium kluyveri. Proc. Natl. Acad. Sci. USA 1945, 31, 373-381. [CrossRef]

29. Bornstein, B.; Barker, H. The energy metabolism of Clostridium kluyveri and the synthesis of fatty acids. J. Biol. Chem 1948, 172, 659-669. [PubMed]

30. Kenealy, W.R.; Waselefsky, D.M. Studies on the substrate range of Clostridium kluyveri; the use of propanol and succinate. Arch. Microbiol. 1985, 141, 187-194.

31. Weimer, P.J.; Stevenson, D.M. Isolation, characterization, and quantification of Clostridium kluyveri from the bovine rumen. Appl. Microbiol. Biotechnol. 2012, 94, 461-466. [CrossRef] [PubMed]

32. Grootscholten, T.; Strik, D.; Steinbusch, K.; Buisman, C.; Hamelers, H. Two-stage medium chain fatty acid (MCFA) production from municipal solid waste and ethanol. Appl. Energy 2014, 116, 223-229. [CrossRef]

33. Wang, Y.-Q.; Zhang, F.; Zhang, W.; Dai, K.; Wang, H.-J.; Li, X.; Zeng, R.J. Hydrogen and carbon dioxide mixed culture fermentation in a hollow-fiber membrane biofilm reactor at $25^{\circ} \mathrm{C}$. Bioresour. Technol. 2018, 249, 659-665. [CrossRef] [PubMed] 
34. Yang, P.; Leng, L.; Tan, G.-Y.A.; Dong, C.; Leu, S.-Y.; Chen, W.-H.; Lee, P.-H. Upgrading lignocellulosic ethanol for caproate production via chain elongation fermentation. Int. Biodeterior. Biodegrad. 2018, 135, 103-109. [CrossRef]

35. Kucek, L.A.; Spirito, C.M.; Angenent, L.T. High n-caprylate productivities and specificities from dilute ethanol and acetate: Chain elongation with microbiomes to upgrade products from syngas fermentation. Energy Environ. Sci. 2016, 9, 3482-3494. [CrossRef]

36. Cardinali-Rezende, J.; Rojas-Ojeda, P.; Nascimento, A.M.; Sanz, J.L. Proteolytic bacterial dominance in a full-scale municipal solid waste anaerobic reactor assessed by 454 pyrosequencing technology. Chemosphere 2016, 146, 519-525. [CrossRef] [PubMed]

37. Wang, Z.; Elekwachi, C.; Jiao, J.; Wang, M.; Tang, S.; Zhou, C.; Tan, Z.; Forster, R.J. Changes in metabolically active bacterial community during rumen development, and their alteration by rhubarb root powder revealed by 16s rRNA amplicon sequencing. Front. Microbiol. 2017, 8, 159. [CrossRef] [PubMed]

38. Kim, E.; Lee, J.; Han, G.; Hwang, S. Comprehensive analysis of microbial communities in full-scale mesophilic and thermophilic anaerobic digesters treating food waste-recycling wastewater. Bioresour. Technol. 2018, 259, 442-450. [CrossRef] [PubMed]

39. Bélaich, J.-P.; Bruschi, M.; Garcia, J.-L. Microbiology and Biochemistry of Strict Anaerobes Involved in Interspecies Hydrogen Transfer; Springer Science \& Business Media: Berlin/Heidelberg, Germany, 2012; Volume 54.

40. Liu, Y.; He, P.; Shao, L.; Zhang, H.; Lü, F. Significant enhancement by biochar of caproate production via chain elongation. Water Res. 2017, 119, 150-159. [CrossRef] [PubMed]

(C) 2019 by the authors. Licensee MDPI, Basel, Switzerland. This article is an open access article distributed under the terms and conditions of the Creative Commons Attribution (CC BY) license (http://creativecommons.org/licenses/by/4.0/). 